\title{
Section thématique
}

\section{Vivre ensemble avec la terre : Conclure et honorer les traités avec les peuples autochtones}

\author{
Sylvie Poirier Université Laval \\ Clinton N. Westman University of Saskatchewan
}

\section{Introduction}

Le Canada est fondé sur un acte de partage d'une générosité presque inimaginable. Les peuples autochtones ont partagé leur nourriture, leurs techniques de chasse et d'agriculture, leurs savoirs pratiques, leurs routes commerciales et leurs connaissances géographiques avec les nouveaux arrivants nécessiteux

James Tully (2008, 244-245).

(...) nos obligations issues des traités sont des engagements solennels, et non des options politiques

Michael Asch (2014, 164).

Notre souveraineté ne découle pas d'un document. Notre souveraineté repose sur une multitude de relations saines, responsables et respectueuses avec toutes nos relations

Leanne Betasamosake Simpson (2015, 22).

Depuis les premiers contacts jusqu'au contexte actuel, les traités, y compris les complexes processus de leur négociation et de leur mise en œuvre, ont non seulement été au cœur des relations entre les peuples autochtones et l'État canadien, mais constituent une part importante de la fondation de ce dernier. Une réalité d'ailleurs que peu de non-Autochtones au Canada (re)connaissent, comme le souligne si justement James Rodger Miller (2009) dans sa rétrospective historique des traités. Au fil des siècles et des régimes politiques, incluant le régime colonial, ces traités ont pris diverses formes. Les traités commerciaux du $17^{\text {ième }}$ siècle seront suivis au $18^{\text {ième }}$ siècle des traités de paix et d'amitié entre nations souveraines, dont le traité de Niagara (1764) est probablement le plus éloquent exemple ${ }^{1}$. Dès le début du $19^{\text {ième }}$ siècle, devant la progression des colons, le besoin de terres et l'avènement d'un régime colonial, de nombreux traités historiques seront négociés et signés, incluant les onze traités numérotés (1871 à 1921). Les peuples autochtones auront ensuite à assumer le choc de la Loi sur les Indiens, de la «mise en réserves " et des écoles résidentielles avant une nouvelle résurgence et mobilisation dans les années 1960 (Alfred 1999 ; 2005 ; Coulthard 2014 ; Kapesh 1976 ; 1979 ; Manuel et Derrickson 2017). Puis, en 1975, c'est la négociation et la signature du premier traité moderne avec la Convention de la Baie James et du Nord québécois (CBJNQ) entre la nation crie, les gouvernements canadien et québécois. Les traités modernes, ainsi que les modalités de leur négociation et de leur mise en œuvre, sont toujours en vigueur aujourd'hui, notamment dans le cadre de la Politique des revendications territoriales globales (1976) ${ }^{2}$.

$\mathrm{Au}$ Canada, de toutes les formes de relations entre les Autochtones, l'État et les non-Autochtones, les relations de traités ont donc été présentes depuis le début. D'est en ouest et du nord au sud, la nature et les formes de ces relations de traités, ainsi que leur incidence sur le rapport au territoire, continuent de varier, selon les régions et les particularités historiques, comme en témoignent d'ailleurs les contributions du présent numéro. Or, dans la très grande majorité des cas, et ce depuis les premières négociations commerciales jusqu'aux traités modernes d'aujourd'hui, les peuples autochtones n'ont jamais dérogé de leur position initiale. Celle selon laquelle ils sont disposés à partager le territoire à la condition de maintenir leur souveraineté, leur autodétermination politique et territoriale, et donc leur identité et leur dignité. Cette position a certes été mise à mal par le régime colonial et l'usurpation de leurs terres. Il n'empêche que plus de 250 ans après le traité de Niagara, suite aux violences, aux souffrances, aux ruptures, aux dépossessions et surtout aux trahisons des gouvernements à l'égard des traités, les peuples autochtones maintiennent leurs revendications, celles de la reconnaissance d'une juste place et d'une autodétermination politique et territoriale. Malgré les revers et les violences subies, ils ont toujours fait preuve de persistance, de résistance et d'imagination politique et culturelle (Poirier 
2010), n'ayant eu de cesse d'adapter leurs revendications aux politiques, discours et idéologies de l'État et de la société dominante, ré-imaginant les termes de leurs relations avec l'État et la société dominante. Depuis les tous débuts et dans ces luttes qui perdurent avec les différents paliers de gouvernements (fédéral, provincial, municipal) afin de faire reconnaitre leurs titres et leurs droits, dans ces dialogues de sourds ou ces faux dialogues (Tully 2016) que sont souvent les processus actuels de négociation, le principal allié des peuples autochtones a très certainement été le territoire. Il demeure leur plus fidèle partenaire.

Dans la perspective de l'État canadien et de son appareil juridique, la question initiale au fondement des traités est la suivante : comment concilier la préexistence des sociétés autochtones avec la souveraineté de la Couronne ? ${ }^{3}$ Une telle formulation peut certes être contestée, voire inversée, comme l'ont d'ailleurs suggéré quelques auteurs, dont Michael Asch (voir Asch 2014 ; Nadasdy 2017, 59) ${ }^{4}$. Elle s'énoncerait alors ainsi : Comment (ré)concilier la souveraineté de l'État canadien avec la souveraineté préexistante et actuelle des peuples autochtones ? Quoiqu'il en soit, de cette question, déjà d'une inextricable complexité, ne serait-ce que par le manque de consensus sur la définition même du concept de "souveraineté ", en découlent plusieurs autres : comment (re)penser la coexistence et (ré)imaginer les relations de traités dans le respect des projets de vie des uns et des autres ? Comment, sur les plans ontologique, politique, juridique et culturel, (ré)concilier l'écart entre les conceptions autochtones et non-autochtones du territoire ? Ces différentes conceptions donnent lieu à leur tour à des compréhensions différentes des termes centraux de la modernité politique mais aussi des traités, comme ceux de souveraineté, d'autonomie, de gouvernance, de pouvoir, d'autorité, de propriété, de frontière et bien sûr de territoire ${ }^{5}$. En cela, les revendications autochtones représentent une invitation inédite à « provincialiser » (Chakrabarty 2009) les termes de la modernité politique, à repenser les bases de la rencontre et du dialogue, et ainsi à envisager une réelle décolonisation (Alfred 2005 ; Coulthard 2014).

Ce rôle central des traités dans la fondation et l'histoire de l'État canadien et la persistance des revendications autochtones expliquent aisément que plusieurs disciplines des sciences sociales et humaines se soient penchées sur ces réalités et enjeux. Depuis au moins les années 1970, et de manière accélérée depuis le début des années $2000^{6}$, toutes ces questions, réflexions et débats autour des traités et des relations de coexistence, de reconnaissance et de réconciliation entre les peuples autochtones, l'État canadien et la société majoritaire ont fait l'objet d'une vaste littérature par des auteurs autochtones (en nombre grandissant) et non-autochtones (Alfred 1999 ; 2005 ; Asch 1997 ; 2014 ; Asch, Borrows et Tully 2018 ; Borrows 2010 ; 2019 ; Borrows et Coyle 2017 ; Clammer, Poirier et Schwimmer 2004 ; Coulthard 2014 ; Davis 2010 ; Eisenberg et al. 2014 ; Hill 2008 ; Manuel et Derrickson 2017 ; Simpson 2008, pour n'en nommer ici que quelques-uns). Dans le contexte néocolonial et néolibéral du Canada d'aujourd'hui, on peut à juste titre se demander dans quelle mesure et de quelle manière les traités contribuent à accroitre le pouvoir, l'autodétermination et l'identité des peuples autochtones ou au contraire à accroître leur aliénation, leur bureaucratisation, leur assimilation et leur dépossession (voir, parmi de nombreux autres, Nadasdy 2003 ; 2017 ; Samson 2016). Cette « double contrainte» (double bind), pour reprendre l'expression de Gregory Bateson, représente certes pour les peuples autochtones un défi et une vigilance de tous les instants. Ceci d'autant plus qu'ils ont appris, au fil d'une expérience plus que centenaire et trop souvent à leurs dépens, que " la parole du Blanc» et sa « bonne foi » (Morales 2017) sont loin d'être fiables. Pourtant, les Autochtones continuent de faire face à ces défis en ré-imaginant, à chaque génération et en prenant appui sur les enseignements de leur tradition orale, les termes de leur engagement avec l'État et la société majoritaire, et, bien entendu, avec et envers le territoire.

Devant la complexité qu'engendrent les relations de traités sur les plans juridique, politique, ontologique et culturel, devant aussi la riche et abondante littérature, savante et critique, et ce toutes disciplines confondues, sur les traités au Canada et sur la nécessité de (re)penser les relations entre l'État et les peuples autochtones, la contribution d'ensemble de ce numéro d'Anthropologica apparait certes modeste, mais est néanmoins tout à fait pertinente. L'un de ses principaux apports consiste à réunir dans un même champ d'analyse des chercheurs travaillant au Québec et au Canada anglophone et parmi des Premières Nations parlant le français et l'anglais comme seconde (ou première) langue.

Les auteurs y adoptent une perspective résolument ethnographique et anthropologique. Les deux rédacteurs invités et les auteurs non-autochtones (Scott, Éthier, Thom, Tipi et Asch) entretiennent des relations de longue date et de collaboration étroite avec les Premières Nations concernées. En prenant appui sur une Première Nation et son territoire, chaque contribution montre comment les relations de traités se déploient sur le territoire, mais aussi comment elles s'enchevêtrent avec le territoire, avec les gouvernements (fédéral et provincial) et entre Premières Nations voisines. Les quatre cas ici présentés, trois au Québec et un en 
Colombie-Britannique, offrent des démonstrations éloquentes de ce que les territorialités autochtones aujourd'hui sont autant de «territorialités enchevêtrées » (Dussart et Poirier 2017) engendrées par la coexistence, souvent difficile et conflictuelle, des régimes de valeurs, des ordres juridiques et des modes de tenure foncière occidentaux (et étatiques) et autochtones. Ces territorialités enchevêtrées découlent également des traités et sont engendrées notamment par les chevauchements des territoires et des négociations.

Le titre du numéro, "Vivre ensemble avec la terre » est inspiré du concept cri, witaskewin, énoncé par les aînés dans leur lecture des termes des traités historiques - particulièrement les traités $4,6,8$ et 10 (Cardinal et Hildebrant 2000, 39 ; voir aussi Asch 2014). Alors que Cardinal et Hildebrant ont traduit witaskewin par « living together on the land " (vivre ensemble sur la terre), nous avons légèrement transformé cette formule en " vivre ensemble avec la terre " afin de rendre l'idée que le territoire est un partenaire et un agent actif dans les relations de traités.

Ce mot - en réalité un "assemblage de concepts " (Sapir 1921, 244) - de la langue des Cris des Plaines est assurément « un petit poème imagiste » (Sapir 1921, 244), avec des implications politiques, philosophiques et cosmologiques. Il convient donc de discuter un peu plus en détail de la retraduction que nous en avons faite. ${ }^{7} \mathrm{La}$ terre (askiy) est le lieu où les gens peuvent se regrouper pour vivre ensemble les uns avec les autres et avec la terre elle-même.

On peut en faire l'analyse grammaticale suivante :

[wi-t-askiy-ew-win]

wi- (particule : « avec»)

-t- (infixe pour maintenir le flux et l'altération entre les sons de consonnes et de voyelles)

askiy («terre»)

-ew- (forme verbale de la troisième personne)

-win (suffixe nominalisateur ; grammaticalement, un mot

se terminant par -win est toujours un nom inanimé)

En effet, la terre est, elle aussi, partie prenante («wi-») de la relation. Comme mentionné précédemment, il ne s'agit pas d'animéité, du moins grammaticalement parlant (voir Wolfart et Ahenakew 1998), mais néanmoins d'une affirmation ontologique. Par le biais du calumet et d'autres médiateurs sacrés, la terre devient partenaire et témoin du traité.

Les aînés cris de la Saskatchewan qui ont servi d'informateurs à Cardinal et Hildebrandt ont insisté sur le fait qu'il s'agit là du terme approprié pour parler de la relation de traité. Witaskewin est un engagement relationnel, et non une chose qui a été réglementée, inspectée ou donnée. C'est une relation authentique avec la terre entière et véritable qui permet des déclarations d'autorité politique complètes et véridiques et des échanges réciproques légitimes. De telles aspirations ont été exprimées le plus souvent - et avec le plus de succès - dans un langage de promesses de traité.

\section{Réflexions sur les traités historiques}

En 2017, lors de la conférence annuelle de la CASCA, les auteurs de cet essai ont co-organisé un panel de sessions multiples, auquel plusieurs des contributeurs de ce numéro ont participé. Si les premières sessions de ce panel ont été l'occasion de discussions approfondies sur les traités historiques et sur la mise en œuvre des traités au Canada (avant et après la Confédération) et en Nouvelle-Zélande, nos contributeurs se concentrent pour la plupart sur la négociation ou la mise en œuvre des traités modernes. En effet, les seuls articles abordant ici les traités historiques sont ceux de Thom et Asch.

Dans de nombreuses régions du Canada, les droits à la terre et aux moyens de subsistance, tels qu'ils sont garantis par les traités historiques, constituent un point de référence important pour les discussions contemporaines autour de l'engagement envers la terre. Par exemple, les Traités numérotés constituent une pierre de touche majeure de l'identité et des aspirations politiques des Premières nations des provinces des Prairies canadiennes. Renforçant le lien ontologique avec la politique historique autant qu'avec la politique contemporaine, le Traité lui-même est sacralisé par un rituel et reconnu par les membres des Premières nations comme une entente sacrée qui permet de nouer des relations de parenté fictives et de partager la terre.

Dans les provinces des Prairies, tout comme dans d'autres régions où des traités historiques ont été signés (peut-être surtout les traités numérotés), le terme « Traité » est souvent utilisé pour revendiquer une identité (Voir Figure 1). De manière générale, «Indien visé par un traité » (Treaty Indian) demeure une catégorie importante dont la signification recoupe celle de catégories comme "Indien inscrit » (Status Indian) selon la Loi sur les Indiens. Elle s'emploie en opposition non seulement aux Euro-Canadiens, mais aussi à des catégories de peuples autochtones telles que "Indiens non visés par un traité » (Non-treaty Indians, qui désigne généralement les membres des Premières nations provenant de l'extérieur de la région), "Indiens non-inscrits » (Nonstatus Indians), et en particulier les Métis. Si les frontières entre le statut de Première nation et celui de Métis se sont avérées flexibles à certaines périodes ou dans certains contextes (par exemple, dans le nord de l'Alberta, lors de la signature du Traité numéro 8 et des premières adhésions à celui-ci), les catégories « Métis » et «Indiens 
non-inscrits " n'étaient, par définition, pas parties aux traités historiques ${ }^{8}$. Tout autochtone qui signait un traité prenait l'identité juridique «d'Indien visé par un traité/ Indien inscrit », devenant ainsi lisible pour l'État. Historiquement, il est arrivé que les Autochtones qualifient ce processus de « devenir Traité » (voir, par exemple, Bella Beaver, citée dans Reddekopp 1997, 18). De telles affirmations identitaires restent importantes aujourd'hui, comme en témoignent les locutions « Je suis Traité » ou « Je suis un Traité ». Dans d'autres régions, l'accent peut être mis sur le statut légal d'Indien plutôt que sur l'affiliation ou l'absence d'affiliation à un traité. Les affirmations de ce genre soulignent l'importance des ententes historiques, qui reconnaissent aux individus contemporains une place sur la terre et servent de principe organisateur pour leurs orientations et aspirations politiques et sociales.

Les traités et ententes conclus entre les peuples autochtones et le Canada, la France, la Grande-Bretagne et/ou les États-Unis s'inscrivent dans une trajectoire historique plus longue, dans la mesure où ces peuples concluaient déjà des pactes ou ententes sacrés entre eux. Comme le déclarait Les Healy, membre de la tribu des Blood (Kainaiwa), à la Commission royale sur les peuples autochtones :

Le concept de traité, inaistisinni, n'est pas nouveau pour la tribu des Blood Inaistisinni est un ancien principe de droit invoqué à plusieurs reprises par les

\begin{abstract}
Blood pour régler des conflits, faire la paix, établir des alliances ou des relations commerciales avec d'autres nations, telles que les Crow, les Gros Ventres, les Sioux et, plus récemment, les Américains en 1855 et les Britanniques en 1877. Inaistisinni est un aspect essentiel du droit immémorial qui a servi à nouer des relations avec d'autres nations. Inaistisinni est un pacte sacré, une entente solennelle ; c'est la forme d'entente véritablement la plus élevée, contraignante à vie pour les parties. Ce traité est si solennel qu'il s'articule autour de l'une de nos cérémonies et de l'un de nos symboles les plus sacrés, le Calumet. (CRPA 1996, II, ch. 2, s. 3.3 ; cité dans Asch 2014, 75)
\end{abstract}

Pour la tribu des Blood et les autres Pieds-Noirs, cette entente solennelle ou pacte sacré servait à refléter les lois naturelles et à créer des relations de parenté fictive entre anciens adversaires, ainsi qu'à réaffirmer la parenté avec la terre elle-même.

Comme le déclarait le chef des Pieds-Noirs (l'actuelle nation Siksika), Pied-de-corbeau, lors de la signature du Traité numéro 7 en 1877 à Blackfoot Crossing :

Pendant que je parle, soyez bons et patients. Je dois parler à mon peuple qui est nombreux et qui s'en remet à moi pour suivre cette voie qui, dans le futur, devra lui être bénéfique. Les plaines sont grandes et vastes. Nous sommes les enfants des plaines ; c'est

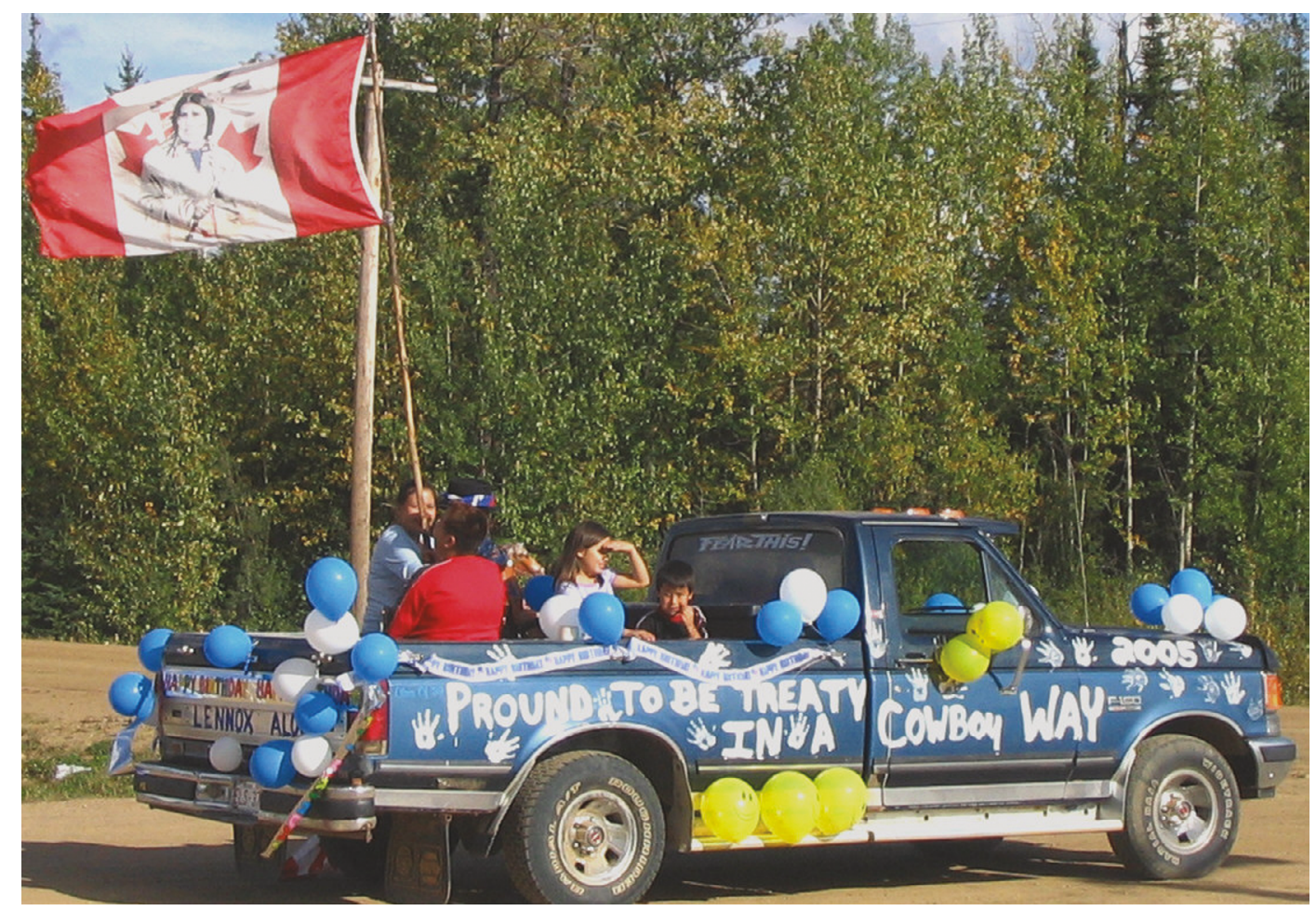

Figure 1: "Proud to be Treaty in a cowboy way "(Clint Westman 2005). 
notre demeure, et le bison a toujours été notre nourriture. J'espère que vous considérerez les Pieds-Noirs, les Blood et les Sarcis comme vos enfants maintenant, et que vous serez indulgent et charitable envers eux. Ils veulent tous que je parle maintenant en leur nom, et j'ai bon espoir que le Grand Esprit saura mettre de la bonté dans le cœur des hommes... Les conseils qui nous ont été donnés, à moi et à mon peuple, se sont avérés très bons. Si la police n'était pas venue dans ce pays, où serions-nous tous maintenant? Les hommes sans scrupules et le whisky nous tuaient si vite que très peu d'entre nous, en vérité, auraient survécu jusqu'à aujourd'hui. La police nous a protégés comme les plumes de l'oiseau le protègent des gelées de l'hiver. Je leur donne ma bénédiction, et je sais que nos cœurs seront dorénavant plus généreux. Je suis satisfait. Je signerai le traité. (Morris 1880, 272 ; cité dans Dempsey et Dempsey 2014, 322)

Pied-de-corbeau fait ici référence à la relation qu'entretient la Confédération des Pieds-Noirs avec la terre («enfants des plaines») et à la nécessité d'une relation de parenté fictive ( « comme vos enfants ») avec la Couronne et les nouveaux arrivants. En usant de comparaisons avec le monde naturel (avec les troupeaux de bisons et les plumes d'hiver de l'oiseau), Pied-de-corbeau explique que le traité constitue un engagement à protéger et à subvenir aux besoins des enfants du souverain. Si d'autres dirigeants se sont montrés plus sceptiques que Pied-decorbeau quant à la possibilité d'établir des relations de parenté rituelle avec les nouveaux arrivants, comme ce fut le cas lors des négociations du Traité numéro 8, de telles références sont restées courantes tout au long de la période de négociation des traités numérotés, en partie parce que les commissaires aux traités eux-mêmes utilisaient ces expressions pour rendre compte de leurs intentions.

Il est intéressant de noter que Pied-de-corbeau et les autres dirigeants évoquaient rarement les cessions de terres dans leurs remarques. Il existe un fossé important entre les promesses orales (mentionnées dans les traditions orales historiques et dans certains documents annexes aux traités ayant pour objet une relation de partage entre parents fictifs) et le texte écrit des traités qui porte sur les aspects juridiques de la cession des terres. En effet, parallèlement à la signature des traités numérotés, le Canada élaborait et mettait en œuvre la Loi sur les Indiens, créant ainsi un régime juridique particulièrement punitif dans les parties méridionales des provinces des Prairies, et qui, à d'importants égards, va encore aujourd'hui à l'encontre de la relation de traité. En outre, les lecteurs contemporains qui connaissent les relations entre la police et les Autochtones dans l'ouest et le nord du Canada pourraient être surpris par l'évaluation positive que fait Pied-de-corbeau de la police. Or, cette conception de la police en tant que bras le plus important de la Couronne est tout à fait cohérente avec les conceptions critiques actuelles de l'État et de son monopole de la violence. La police et l'application de la loi restent les domaines où la réconciliation et la pensée relationnelle doivent être le plus urgemment mises en œuvre.

Parvenir à une compréhension commune de «l'esprit véritable et l'intention originelle » des traités est un problème politique urgent avec des implications majeures pour l'utilisation des terres et la réconciliation (Treaty 7 Tribal Council et al. 1996 ; cf. Cardinal et Hildebrandt 2000). Ainsi, deux des questions clés dans les relations de traité contemporaines portent sur les indemnisations et les consultations afférentes aux activités industrielles autorisées sur les terres de la Couronne où les Premières nations visées par un traité détiennent des droits de chasse, de pêche et de cueillette. A partir de 1982, ce sont les tribunaux qui ont servi de lieu privilégié pour de telles discussions sur la signification des droits issus des traités hors réserve.

Comment les Premières nations visées par un traité ont-elles mobilisé les promesses de traité pour obtenir la juridiction et le pouvoir législatif sur leurs propres terres (c.-à-d. dans les réserves) ainsi que sur leurs citoyens ? Les progrès ont été moins importants dans ce domaine. Dans certaines juridictions (par exemple, dans les Territoires du Nord-Ouest) les nations signataires de traités historiques ont longtemps été considérées comme des partenaires de négociation admissibles aux traités modernes ; dans d'autres juridictions, cela n'a généralement pas été le cas ${ }^{9}$. Les négociations sur les revendications particulières, sans doute dans une moindre mesure que celles sur les revendications globales, ont le potentiel d'aboutir à l'octroi de terres, de ressources et d'une nouvelle reconnaissance politique aux Premières nations, souvent en raison de leur insistance sur les traités historiques comme fondement des relations et engagements contemporains. C'est le cas de certaines revendications relatives aux droits fonciers issus des traités, comme par exemple la série de demandes d'adhésion au Traité numéro 8 effectuées dans le nord de l'Alberta depuis les années 1990 ; les montants des indemnisations en espèces, en terres ou en infrastructures octroyés récemment dans le cadre du règlement de ces revendications peuvent rivaliser avec ceux octroyés dans le cadre du règlement de certaines revendications globales (Westman 2017b). D'autres revendications particulières, alléguant la cession injustifiée de terres de réserve à des fins de colonisation agricole (notamment celles des Kainaiwa et des Siksika), pourraient également faire l'objet d'une indemnisation très importante, que ce soit dans le cadre d'un règlement 
ou devant les tribunaux. Or, là encore, le règlement de telles revendications particulières ne devrait conférer aucun nouveau pouvoir législatif aux Premières Nations.

Il existe des mécanismes non contraignants qui permettent d'exprimer et de clarifier les significations contemporaines des relations de traité, comme par exemple le Bureau du commissaire aux traités en Saskatchewan ou les discussions bilatérales sur les traités, largement au point mort, en Alberta. Il n'empêche que les processus de revendication et les tribunaux semblent avoir été les mécanismes les plus importants sur le plan politique.

Pour comprendre les traités historiques, il convient de reconnaître l'écart entre le texte écrit et les promesses orales qui l'accompagnent et de prendre en compte les promesses des tribunaux dans l'interprétation du texte. En adoptant une perspective de traité sur les questions relatives aux terres et aux droits historiques, et en réfléchissant de manière relationnelle aux événements en cours dans les territoires couverts par les traités historiques, il devient possible d'envisager de nouvelles façons de vivre ensemble les uns avec les autres et avec la terre. Quoi qu'il en soit, la réalisation des promesses des traités historiques constitue encore aujourd'hui un défi pour la politique.

\section{La vie sociale des traités : Pour une approche relationnelle des traités}

Par définition, un traité est une entente entre peuples/ nations souveraines afin de régler un conflit, restaurer la paix et sceller une alliance, parmi d'autres objectifs. Dans le cas des peuples autochtones dans des colonies de peuplement comme le Canada, les traités visent à en arriver à une entente pour la coexistence sur le territoire et le partage de celui-ci, une entente qui permettrait aux peuples autochtones d'élaborer et de mettre en œuvre leur propre projet de société et d'exercer une forme d'autonomie et d'autodétermination politique et territoriale. Pour plusieurs Autochtones et non-Autochtones, les traités entre l'État canadien et les peuples autochtones peuvent offrir une «base éthique relationnelle " pour ce vivre ensemble avec la terre (voir Asch 2014). Aaron Mills (2017) présente la conception anishinaabe des traités comme « une avenue relationnelle complète » (a total relational means). Il écrit :

Les traités ne sont pas des instruments juridiques ; ce sont des cadres pour des relations justes : l'ensemble des moyens relationnels par lesquels nous nous orientons et réorientons les uns vers les autres au fil du temps, afin de bien vivre ensemble et avec toutes nos relations au sein de la création. (...) ils sont à la fois politiques, sociaux, économiques, spirituels et écologiques. Ils sont ce qui nous permet de nous constituer en communautés de communautés, au-delà de nos différences. (Mills 2017, 225) ${ }^{10}$

D'autres Premières Nations et auteurs autochtones se reconnaissent très certainement dans cette perspective anishinaabe; une perspective que partagent aussi de nombreux chercheurs non-autochtones.

Plusieurs auteurs s'entendent donc à reconnaitre que les traités, historiques et modernes, entre les gouvernements et les Premières Nations ne peuvent être réduits à des instruments légaux ou à une forme de contrat, mais doivent être considérés plutôt comme une alliance politique, voire comme un pacte sacré entre peuples et comme un engagement moral et éthique des uns envers les autres, mais aussi envers le territoire, les autres qu'humains, les ancêtres et les générations futures. Or, cette conception se heurte à celles véhiculées et mises de l'avant par les gouvernements et la société majoritaire. Les positions et perspectives divergentes entre les peuples autochtones et l'État canadien concernant les traités sont très bien résumées par Martin Papillon et Audrey Lord $(2013,345)$ : "Alors que les peuples autochtones y voient le fondement d'une nouvelle relation avec l'État, les gouvernements conçoivent encore aujourd'hui ces ententes d'abord comme une transaction foncière permettant de clarifier, et surtout de limiter, la portée des droits ancestraux sur le territoire».

$\mathrm{Au}$ Canada, les traités historiques et modernes, leur énonciation, les processus de leur négociation et de leur mise en œuvre, ont toujours été traversés et animés par des conflits d'interprétation, mais aussi par des conflits d'ordre ontologique. En effet, les groupes en présence ont des conceptions différentes à la fois du «traité » et $\mathrm{du}$ « territoire ». Dans les processus de communication et de négociation, nous assistons à ce que Viveiros de Castro nomme « une équivoque non-contrôlée » (cité dans Blaser 2009). En d'autres termes, les groupes en présence ne parlent pas de la même chose (le traité et le territoire), mais ne le savent pas. Louvrage de Asch (2014) sur les traités numérotés en donne un exemple éloquent. Les traités semblent condenser à eux seuls tous les « écarts » (dans le sens que lui accorde François Jullien ${ }^{11}$ ) entre les conceptions occidentales et autochtones de l'esprit d'un traité, de ce qu'implique une alliance entre peuples souverains, du territoire et des relations avec celui-ci, mais aussi de la signification de concepts clé tels ceux d'autonomie, de pouvoir ou d'autorité (Voir Éthier, Ottawa et Coocoo dans ce numéro). À tous ces niveaux, les divergences conceptuelles (et ontologiques) sont flagrantes et apparaissent même souvent comme incommensurables 
et irréconciliables. Pourtant, à travers le Canada, des négociations sont en cours et des ententes sont signées. Alors que certains sont confiants dans la pertinence des traités pour rétablir une certaine justice pour les peuples autochtones dans le respect de leur différence, d'autres sont plus pessimistes ou du moins émettent de sérieuses mises en garde, et probablement avec raison ${ }^{12}$. Toutefois, ce que les gouvernements et la société majoritaire peinent à (re)connaître est le fait que les peuples autochtones y jouent leur devenir, leur identité et leurs projets de vie ; ils y jouent leur responsabilité envers le territoire, envers les ancêtres et les générations futures. Pour cela, ils sont prêts à s'astreindre à des processus de négociation longs, ardus et souvent frustrants (Éthier, Ottawa et Coocoo ; et Tipi et Boivin dans ce numéro), et à faire face à des violences d'ordre structurel et ontologique (Scott et Thom dans ce numéro).

À la suite de Asch (2014) et Mills (2017) (cités cihaut), il nous semble ici pertinent d'explorer plus avant ce qu'implique une approche relationnelle des traités. Premièrement, à l'instar des objets (Appadurai 1988), les traités ont une vie sociale et génèrent une multiplicité de significations, d'interprétations, d'attentes et d'espoirs. Les traités ont une vie sociale dans la mesure où ils sont porteurs de qualités, de capacités et de potentialités relationnelles qui interpellent et engagent les humains, les institutions et le territoire. Une vie sociale qui met ainsi en co-présence et donc en dialogue les régimes de valeurs et d'historicité autochtones et non-autochtones. Cette vie sociale d'un traité s'exprime et se déploie à différentes étapes, et ce de manière diachronique : les processus de négociation du traité ; l'énonciation et les termes du traité ainsi que l'esprit du traité; les dynamiques de mise en œuvre du traité sur le long terme; les modifications et amendements apportés au traité, incluant les termes de résolutions de conflits issus du traité; etc. (voir aussi Coyle 2017). Chacune de ces étapes mobilise le temps, l'énergie et les expertises d'un ensemble d'acteurs autochtones et non-autochtones. Une fois signés, les traités deviennent un acteur incontournable dans les relations entre les gouvernements, les industries, les Autochtones et le territoire; ils jouent un rôle clé dans l'actualisation, la transformation et le déploiement de ces relations. Une fois ratifiés, les traités sont un point de départ d'une relation et non une fin en soi (Papillon et Lord 2013, 345 ; voir aussi Scott dans ce numéro). Leur devenir et leur déploiement sont truffés de potentialités, d'incertitudes et d'indétermination. Alors qu'une telle perspective est pour le moins inconfortable pour les modernes (et l'État), habitués à fonctionner sur le mode du contrôle et de la certitude, elle s'accorde bien avec les ordres relationnels politique et ontologique des Autochtones ${ }^{13}$.

Dans un deuxième temps, une approche relationnelle des traités doit considérer le territoire, non pas comme une entité passive, mais plutôt comme un acteur à part entière dans le processus de négociation et de mise en œuvre du traité. C'est en ce sens que Clinton Westman souligne à propos du Traité numéro 8: "Lesprit de la terre devient un partenaire et un témoin du traité » (Westman 2017a). Cette dimension met à jour un conflit ontologique majeur entre les conceptions occidentales et autochtones du territoire. Les Autochtones entretiennent avec le territoire et les autres qu'humains qui l'habitent une relation de réciprocité, de partage et de responsabilité, une relation conçue sur le long terme selon le principe de l'ancestralité et sur la base d'une ontologie relationnelle (Poirier 2013 ; Scott 2017 et dans ce numéro) ou une « ontology of care » (Pasternak 2017). De son côté, l'État canadien entretient une relation utilitariste avec le territoire considéré comme une surface à exploiter, une relation conçue sur le court terme sur la base d'une ontologie naturaliste. Difficile dès lors de (ré) concilier ces deux conceptions du territoire qui reposent sur des ontologies et des régimes de valeurs et d'historicité divergents ${ }^{14}$.

La troisième dimension d'une approche relationnelle des traités interpelle l'ensemble des non-Autochtones. Une approche relationnelle des traités implique donc le territoire, les peuples autochtones, les institutions et les différents paliers de gouvernement, mais aussi indubitablement tous les non-Autochtones. La question inextricable de la coexistence des souverainetés autochtone et étatique étant loin d'être résolue sur les plans politique, juridique et constitutionnel, elle ne peut évidemment être simplement ignorée ou mise sous le tapis. Nous avons tous entendu l'expression: "We are all treaty people ". Ce constat doit être pris très au sérieux dès lors que l'on reconnait que la présence et les activités des non-Autochtones sur le territoire canadien ont été rendus possible par les traités et par la grande générosité des peuples autochtones, comme le fait remarquer James Tully (2008) dans la citation en exergue de ce texte. Il appert dès lors que les non-Autochtones sont redevables aux peuples autochtones et partie prenante des traités existants et à venir. Jean-Olivier Roy (2018) fait d'ailleurs remarquer :

Les non Autochtones doivent, au même titre que les Autochtones, s'engager dans une profonde démarche de refonte identitaire quant à leur propre identité coloniale. Cela permettrait de mieux comprendre les enjeux des traités à venir, de les voir comme un gain (sur le plan de leur identité) et non comme une perte 
(économique, territoriale) et à terme, de devenir également citoyen des traités, des ententes conclues et à conclure, et de s'engager à les respecter. ${ }^{15}$

Enfin, il nous semble pertinent de faire une dernière remarque sur les conflits d'interprétation (et les conflits ontologiques) et sur une approche relationnelle des traités. Il s'agit de l'écart, déjà souligné par plusieurs auteurs, entre l'ordre légal et étatique moderne et les ordres juridiques autochtones. Lordre occidental moderne repose d'abord et avant tout sur les droits individuels, auxquels il donne une place prépondérante. Les ordres autochtones reposent, quant à eux, davantage sur les droits collectifs, ceux-ci étant couplés aux devoirs et responsabilités envers le groupe, envers le territoire et les autres qu'humains qui l'habitent, envers les ancêtres, envers les générations qui ont précédé et celles à venir. Ce couple droit/devoir est en réalité inséparable, et l'état de droit des modernes aurait en cela beaucoup à apprendre des ordres autochtones. Cet écart est aussi souligné par Colin Scott (dans ce numéro) lorsqu'il distingue l'ordre institutionnel de l'état et l'ordre relationnel des Autochtones (voir aussi Mills 2017). Cette distinction se répercute dans les façons divergentes de concevoir les traités : comme une opportunité de construire des relations basées sur la coexistence, la responsabilité partagée et le partenariat du côté des peuples autochtones, comme un contrat où prédomine un discours basé sur les « droits » du côté de l'État.

\section{Dans ce numéro}

À travers le Canada, la place que les traités occupent dans la vie des Premières Nations varie sensiblement d'un territoire à l'autre et donne lieu à diverses situations : les Premières Nations signataires d'un traité, soit-il historique ou moderne (Scott; Thom dans ce numéro) ; les Premières Nations engagées dans des processus de négociation en vue de la signature d'un traité moderne (Éthier, Ottawa et Coocoo ; Tipi et Boivin ainsi que Thom dans ce numéro) ; les Premières Nations qui, pour diverses raisons, refusent de s'engager dans de tels processus et empruntent d'autres avenues politiques et juridiques pour faire valoir leurs droits inhérents (Pasternak 2017) ; les Premières Nations sans traité mais voisines de signataires de traités (Éthier, Ottawa et Coocoo ; et Thom dans ce numéro). À cela s'ajoute les nombreuses situations de chevauchements des territoires et des négociations (Éthier, Ottawa et Coocoo ; et Thom dans ce numéro). Tout cela contribue à créer, particulièrement sur les territoires et dans la vie des Premières Nations concernées, des situations complexes, difficiles et souvent conflictuelles. Sur les territoires ancestraux et revendiqués, les Premières Nations n'ont jamais évolué en vase clos, mais se sont constamment engagées, au fil des générations et des conditions changeantes, à reformuler et à (ré)imaginer leurs interactions et leurs relations avec l'État et ses institutions, avec les Premières Nations voisines, et avec les non-Autochtones et les industries ayant des intérêts sur lesdits territoires.

Concernant les relations de traités, chacune des quatre contributions offre un exemple probant de la complexité des territorialités enchevêtrées actuelles, de la coexistence des ordres étatique et autochtones, et de la dynamique de ces derniers, notamment en ce qui concerne les systèmes de tenure foncière, la question des délimitations territoriales, les activités de chasse, trappe, pêche et cueillette, les processus de transmission des savoirs et des territoires, ou encore les formes d'autorité et de pouvoir. Le numéro offre aussi quelques exemples contrastés. Ainsi, à l'époque où les Cris de Eeyou Istchee (Scott dans ce numéro) signaient la CBJNQ, il y a de cela plus de 40 ans, deux nations voisines, les Atikamekw Nehirowisiwok (Éthier, Ottawa et Coocoo dans ce numéro) et les Innu (Tipi et Boivin dans ce numéro) entamaient, quant à elles, un long processus de négociation dans le cadre de la Politique des revendications territoriales globales, sans avoir conclu d'ententes finales à ce jour.

Dans sa contribution, Scott nous donne un exemple éloquent de la vie sociale d'un traité en constant devenir. Son engagement de longue date avec les Cris de Eeyou Istchee lui offre une perspective inédite. Scott démontre comment, depuis la signature de la CBJNQ, les Cris de Eeyou Istchee ont non seulement gagné en autonomie politique et économique, mais n'ont eu de cesse de négocier, soit des amendements au traité initial, soit des ententes complémentaires. Au Canada, l'exemple des Cris de Eeyou Istchee est certes unique. Signataires du premier traité moderne, ils permettent d'analyser l'évolution des relations de traité au fil des quatre dernières décennies. Loin de se contenter des termes initiaux du traité, et dans l'objectif d'accroitre leur gouvernance sur leur territoire, les Cris, forts d'un nouveau rapport de force, n'ont eu de cesse de maintenir des relations de négociation avec les trois paliers de gouvernement et, à la limite, de faire mentir les objectifs étatiques de " certitude » et de "finalité » souvent associés aux traités. C'est ainsi que depuis la signature de la CBJNQ, explique Scott, ils ont négocié 24 ententes complémentaires.

Sans nier le pouvoir des Cris de Eeyou Istchee, il y a toutefois un revers à cette omniprésence des relations de traités dans toutes les sphères de la vie des Cris, convient Scott. Concernant les relations de traité, il souligne la vigilance constante que les Cris de Eeyou Istchee doivent exercer afin de pallier à la violence structurelle 
et ontologique, présente sous différentes formes, et de maintenir leur ordre relationnel, soit des relations de respect et de réciprocité autant envers les gouvernements et leurs représentants qu'envers le territoire et les autres qu'humains. Face au néolibéralisme et aux impératifs du capitalisme et de la modernité, il s'agit là d'un pari certes difficile à tenir, mais cela vaut pour toutes les Premières Nations. Avec ce traité moderne, et au fil des quarante dernières années, les Cris de Eeyou Istchee ont acquis un pouvoir de négociation et une marge de manœuvre au niveau politique et économique dont ne bénéficient pas encore les Nations Atikamekw Nehirowisiw et Innu, les deux prochains exemples de ce numéro. Lorsque la CBJNQ a été signée sans qu'elles ne soient consultées, ces deux nations voisines ont d'ailleurs vu leurs titres et leurs droits ancestraux éteints sur une partie de leurs territoires.

La Nation Atikamekw Nehirowisiw est pour sa part engagée dans un processus de revendications globales avec les deux paliers de gouvernement depuis la fin des années 1970. Dans leur contribution, Benoit Éthier, Gérald Ottawa et Christian Coocoo présentent un portrait convaincant de la manière dont les Atikamekw Nehirowisiwok abordent ce processus de négociation en faisant preuve d'une " résistance créatrice ». Ils montrent comment la Nation, au fil des décennies, a acquis une plus grande connaissance et assurance dans l'art du dialogue et de la traduction en faisant valoir les concepts atikamekw de pouvoir, de droits et de responsabilités au fondement de leur ordre normatif relationnel et de leur conception du territoire. Les auteurs soulignent que bien que les règles de la négociation aient été édictées par l'État néocolonial, y compris la «clause d'extinction ", le «fardeau de la preuve » ou encore «l'obligation de dette $»^{16}$, l'engagement de la Nation Atikamekw Nehirowisiw dans le processus de négociation n'en contribue pas moins au renforcement de leur identité, de leur relation au territoire et de leur pensée politique. Cette continuité de leur ordre normatif, la « résistance créatrice» dont ils ont fait preuve et les efforts consentis pour le maintien de leurs responsabilités à l'égard du Nitaskinan (territoire ancestral) et des territoires familiaux ainsi que pour la transmission des savoirs peuvent aussi être envisagées comme des défaites de l'État colonial, comme des brèches dans sa propre souveraineté.

La longueur et la difficulté des processus de négociation, les rapports de pouvoir inégaux, les violences d'ordre structurel et ontologique, le manque de « bonne foi » des gouvernements, la bureaucratisation accrue de la gouvernance du territoire, ou encore le fait que le Québec continue d'engranger les profits des ressources (foresterie, hydroélectricité, tourisme) du territoire noncédé des Atikamekw Nehirowisiwok n'ont pas eu raison de la détermination de cette Première Nation. Il en va de même pour la Nation Innue, présentée ici par Sukrän Tipi et Hélène Boivin.

Les contributions d'Éthier, Ottawa et Coocoo, et de Tipi et Boivin témoignent d'une autre réalité, celle à l'effet que le processus même de négociation territoriale, dans lequel les Premières Nations concernées doivent faire la preuve d'une occupation ancestrale du territoire, devient un levier majeur pour la documentation, la valorisation et la transmission des savoirs locaux et de la langue et pour la consolidation des relations intergénérationnelles. Sont alors mobilisés les principaux acteurs locaux, en particulier les familles, les aînés, les écoles et les conseils de bande, dans des initiatives de transmission qui engagent les communautés toute entières.

À l'instar de la Nation Atikamekw Nehirowisiw, la Première Nation des Pekuakamiulnuatsh (Innus du Lac Saint-Jean ou Mashteuiatsh) est engagée depuis plus de 40 ans dans un processus de négociation territoriale, actuellement au sein du Regroupement Petapan ${ }^{17}$. La signature d'un traité moderne est d'ailleurs envisagée à court terme. Dans leur contribution, Tipi et Boivin retracent les grandes lignes de l'occupation ancestrale du territoire par les Pekuakamiulnuatsh ainsi que l'histoire récente du processus de négociation avec les deux paliers de gouvernement. Mais surtout, elles donnent un exemple éloquent du travail colossal consenti par la Première Nation des Pekuakamiulnuatsh dans la documentation de leur territorialité, de leurs savoirs et de leur langue. Parmi les nombreuses initiatives de documentation entreprises au fil des décennies, la plus récente est le projet de recherche multidisciplinaire Peshunakun initié en 2008. Les objectifs, les étapes et la méthodologie du projet Peshunakun, tels que présentés par les auteures, permettent d'apprécier l'investissement humain que représente celui-ci. Laccent est mis ici sur la territorialité des Pekuakamiulnuatsh sous l'angle du lien entre leur langue, le nehlueun ${ }^{18}$, la topographie du territoire, les lieux et les toponymes. Cela met en évidence l'ancrage même de l'identité innu (ou ilnu) dans l'espace. Limportance accordée aux toponymes comme vecteurs de savoirs et de mémoires permet à son tour d'évoquer les ancêtres et d'identifier les territoires familiaux traditionnels de chasse, de trappe, de pêche et de cueillette. Les résultats du projet Peshunakun révèlent une richesse et un potentiel sémantique, historique, mnémonique et identitaire à la limite inépuisable, dans un contexte d'affirmation politique et d'aspiration des Pekuakamiulnuatsh à l'autodétermination via la signature d'un traité.

Le texte de Brian Thom nous conduit sur le territoire des traités des Coast Salish au sud-est de l'île de 
Vancouver. Ce cas offre un exemple des plus significatifs de la complexité légale et politique engendrée par la proximité sociale et géographique de nations signataires et non-signataires et de la question des " chevauchements " (overlapping claims). Thom rappelle que les concepts étatiques de «frontières » fixes et de territoires clairement et durablement délimités ont contribué aux problèmes de chevauchement et à la perpétuation des rapports coloniaux de pouvoir ${ }^{19}$. Sur ce territoire se côtoient des Premières Nations signataires des traités historiques Douglas (1850-1854) et d'autres qui n'ont pas encore signé de traités mais qui sont engagées dans la négociation d'un traité moderne. Dans un tel contexte, l'analyse révèle, de manière probante, les complexes enchevêtrements légaux et socioculturels qui découlent des relations de traités, au niveau des chevauchements territoriaux, des relations intertribales et de la définition et de la mise en œuvre contemporaines des droits issus des traités. Ce texte nous rappelle aussi comment les Coast Salish continuent de mettre en œuvre leurs propres conceptions de la communauté et de la territorialité - lesquelles sont plus extensives et flexibles que ce qu'imposent l'État canadien et la Loi sur les indiens.

À la lecture du texte de Thom, plusieurs questions viennent à l'esprit. Comment les droits issus des Traités Douglas, notamment les droits de chasse et de pêche, de même que leur interprétation ont-ils évolué depuis la signature de ces traités? Comment les cours de justice définissent-elles ces droits à la lumière des revendications des Premières Nations et des principes de leurs systèmes coutumiers? Dans le contexte contemporain, comment (ré)concilier les droits issus des traités et ceux issus des systèmes normatifs traditionnels? La force de l'argumentaire de Thom est de s'appuyer sur quelques exemples concrets de "contrevenants " autochtones traduits en justice et de mettre en dialogue les termes du traité, les principes des ordres politiques et légaux autochtones et leurs interprétations respectives par les juges. Les principes autochtones mis de l'avant par Thom sont les suivants : le partage et la coopération entre les communautés et les Premières Nations; une certaine flexibilité dans l'affiliation et dans l'exercice des droits de chasse et de pêche sur divers territoires; et les réseaux de parenté bilatérale et élargie qui donnent accès à différents territoires. L'État canadien et son système de justice peinent souvent à reconnaitre ces principes dans la mesure où ils s'accordent difficilement aux principes de la modernité politique (frontières fixes, droits individuels, etc.). Pourtant, soutient Thom, s'ils étaient mieux reconnus sur les plans légal et politique, les principes normatifs autochtones de partage et de coopération et leur mise en œuvre pourraient permettre de solutionner une partie des problèmes de « chevauchements » et de l'exercice des droits ancestraux ${ }^{20}$.

\section{Conclusion}

Les contributions de ce numéro thématique montrent comment les peuples autochtones engagés dans des relations de traités, qu'ils aient ou non conclu des ententes, travaillent sur deux fronts simultanément. Celui, d'une part, de négocier et de convenir d'ententes avec les gouvernements afin de faire valoir et respecter leurs droits, et celui, d'autre part, de maintenir leur identité et leur responsabilité envers le territoire, les ancêtres et les générations à venir. Ces deux objectifs demandent une pensée politique mature dans la mesure où, entre autres, ils relèvent d'ordres différents, soit institutionnel et relationnel. Le défi est aussi de tenter d'éviter la bureaucratisation de la relation au territoire selon les termes de l'état et de la société majoritaire, tout en assurant la transmission des savoirs et de la pensée politique aux jeunes générations.

Dans ce combat entre David et Goliath, ceux qui ont le pouvoir et le monopole de la violence, soit les gouvernements coloniaux et les corporations, n'ont pas intérêt à faire preuve d'imagination et d'initiative dans leur pensée et leur action politiques. Plus souvent qu'autrement, il en revient d'ailleurs aux Autochtones de proposer des innovations dans les termes et le déploiement des relations de traités. Dans les processus de négociation et les relations de traités, l'État et la société majoritaire gagneraient à faire confiance aux ordres normatifs autochtones et à reconnaitre leur légitimité. Or, qu'ils soient reconnus ou non par l'État, les ordres autochtones se maintiennent tout en se réinventant, comme le démontrent les contributions du numéro. Cette persistance constitue autant de formes de résistance à la souveraineté canadienne.

Ce manque d'imagination et de volonté politique des gouvernements coloniaux et l'absence d'un « dialogue équitable » entre les parties afin de négocier la réconciliation sur la base d'institutions et de principes renouvelés (Tully 2016, 223-256) ${ }^{21}$ privent tous les citoyens d'un projet de société qui pourrait être novateur et partagé avec les peuples autochtones et le territoire, un projet dans lequel celui-ci ne serait plus traité comme une simple surface à exploiter et à violer, mais comme un partenaire. Les travaux critiques de chercheurs et de penseurs autochtones et non-autochtones visant à repenser les relations entre les peuples autochtones et l'État sur de nouvelles bases alimentent les politiques de l'espoir.

Sylvie Poirier, Département d'anthropologie, Université Laval, Québec, QC, Canada. Courriel: Sylvie.poirier@ant.ulaval.ca. 
Clinton N. Westman, Département d'archéologie et d'anthropologie, University of Saskatchewan, Saskatoon, Saskatchewan, Canada. Courriel: clint. westman@usask.ca.

\section{Notes}

1 Il faisait suite à la Proclamation Royale (1763).

2 Depuis la mise en œuvre de la Politique des revendications territoriales globales, 26 traités modernes ont été conclus. Il y aurait 99 Premières Nations à des tables de négociations à travers le Canada. Aujourd'hui, la plupart de ces " traités modernes » intègrent une autorité législative dans les domaines-clés, soit une entente administrative constitutionnellement reconnue et désignée comme gouvernement autonome.

3 Cette question a été d'abord formulée par le juge Lamer dans Van der Peet (1996) et a été reprise dans Delgamuukw en lien avec l'article 35 (1) (elle a été citée depuis par plusieurs auteurs dont Tully $(2008,224)$ et Asch (2014)) : " la conciliation de la préexistence des sociétés autochtones et la souveraineté de la Couronne »(Chief Justice Antonio Lamer, Delgamuukw 1997, para. 186).

4 Pour Asch, comme pour d'autres, la souveraineté canadienne est illégitime en ce qu'elle s'est appuyée dès le début sur une doctrine coloniale aujourd'hui discréditée, celle du terra nullius (Asch 2002 ; voir aussi Pratt 2004).

5 Sur toutes ces questions, voir aussi l'ouvrage récent de Paul Nadasdy (2017) sur les Premières Nations du Yukon qui ont signé un " traité moderne ".

6 Le rapport de la Commission royale sur les peuples autochtones (1996) ainsi que le rapport de la Commission de vérité et réconciliation (2015) auront certes contribué à cet intérêt renouvelé.

7 Asch et Westman semblent avoir opérationnalisé indépendamment l'un de l'autre le mot tel qu'employé par Cardinal et Hildebrandt, et ce, de manière légèrement différente mais largement compatible (voir par exemple Westman 2016).

8 Historiquement, les questions relatives aux droits et intérêts des Métis ont souvent été réglées par l'intermédiaire de «certificats " octroyés aux individus. Les questions relatives aux terres, aux programmes et aux moyens de subsistance des Métis font toujours l'objet de litiges, malgré une série d'avis prometteurs émis par les tribunaux.

9 Les Métis sont autorisés à signer les traités modernes (là encore particulièrement ceux des Territoires du Nord-Ouest).

10 Sur le paradigme relationnel, voir aussi Starblanket et Stark 2018.

11 François Jullien $(2009,159)$ écrit : «(..) considérer le pluriel des cultures sous l'angle de l'écart les fait paraître comme autant de possibles ouverts, inventifs, dont on peut exploiter la fécondité ".

12 C'est le cas notamment de Colin Samson (2016) pour les Innus du Labrador et de Paul Nadasdy (2017) pour les Premières Nations du Yukon, des Nations ayant signé récemment des traités modernes. Les deux auteurs montrent comment en adoptant/adaptant des formes de gouvernance et de bureaucratie " modernes " ou étatiques via un traité, ce dernier agit en quelque sorte comme un cheval de Troie permettant la poursuite du projet colonial.
13 Rappelons, par exemple, que dans les mondes ontologiques des Cris et de plusieurs Premières nations, les principaux symboles ou métonymes des Traités et autres accords sacrés comprennent le calumet et le tabac. Ceux-ci se voient tous deux accorder le statut d'animéité dans les événements de parole cris, et peuvent ainsi dénoter des rencontres plus qu'humaines.

14 Voir aussi la thèse de Julie Depelteau (2019) sur ces différentes conceptions du territoire à partir d'une analyse approfondie des textes produits par la Nation atikamekw nehirowisiw et les gouvernements (fédéral et québécois) dans le cadre des négociations territoriales.

15 Il ajoute : "La récente mouture du serment de citoyenneté des immigrants les engage à honorer ces traités, alors que la population dominante ne le fait toujours pas. " (Roy 2018). Voir aussi Roy 2019.

16 À ces règles s'ajoute celle de l'imposition de la langue du colonisateur dans tout le processus de négociation, dans ce cas-ci le français, alors que la langue atikamew est encore la langue maternelle de $96 \%$ des Atikamekw Nehirowisiwok.

17 Le Regroupement Petapan rassemble les Premières Nations innues de Mashteuiatsh, Essipit et Nutashkuan et a pour mission la négociation et la signature d'un projet d'entente de traité avec les gouvernements du Canada et du Québec.

18 Une langue qui malheureusement n'est presque plus parlée.

19 Sur le concept de "frontières ", Leanne Betasamosake Simpson écrit : "Pour les nations autochtones, les frontières ne sont pas des lignes rigides sur une carte mais des zones de diplomatie, de cérémonie et de partage accrus » $(2015,19)$.

20 Sur la question des « chevauchements ", l'analyse de Thom rejoint celle de Sylvie Vincent pour les mondes algonquiens (2016).

21 Martin Hébert $(2019,393)$ écrit à ce sujet : " Malgré la présence constante du thème de la réconciliation dans le discours social canadien depuis au moins le milieu des années 1990, nous nous trouvons encore devant un profond déficit d'imagination pour envisager un avenir où il est possible d'agir sur les conditions structurelles qui reproduisent la marginalisation des peuples autochtones ».

\section{Références}

Alfred, Taiaiake, 1999. Peace, Power, Righteousness: An Indigenous Manifesto. Toronto, Oxford University Press.

Alfred, Taiaiake, 2005. Wasase: Indigenous Pathways of Action and Freedom. Peterborough, ON, Broadview Press.

Appadurai, Arjun, 1988. The Social Life of Things: Commodities in Cultural Perspective. Cambridge, Cambridge University Press.

Asch, Michael (dir.), 1997. Aboriginal and Treaty Rights in Canada: Essays on Law, Equality, and Respect for Difference. Vancouver, University of British Columbia Press.

, 2002. «From Terra Nullius to Affirmation: Reconciling

Aboriginal Rights with the Canadian Constitution ". Canadian Journal of Law and Society, 17 (2) : 23-39. https://doi.org/10.1017/S0829320100007237 
_ 2014. On Being Here to Stay: Treaties and Aboriginal Rights in Canada. Toronto, University of Toronto Press.

Asch, Michael, John Borrows, et James Tully (dir.), 2018. Resurgence and Reconciliation: Indigenous-Settler Relations and Earth Teachings. Toronto, University of Toronto Press.

Blaser, Mario, 2009. "The Threat of the Yrmo: The Political Ontology of a Sustainable Hunting Program ». American Anthropologist, 111 (1) : 10-20. https://doi. org/10.1111/j.1548-1433.2009.01073.x

Borrows, John, 2010. Canada's Indigenous Constitution. Toronto, Toronto University Press.

_ 2019. Law's Indigenous Ethics. Toronto, Toronto University Press.

Borrows, John, et Michael Coyle (dir.), 2017. The Right Relationship: Reimagining the Implementation of Historical Treaties. Toronto, University of Toronto Press.

Cardinal, Harold, et Walter Hildebrandt, 2000. Treaty Elders of Saskatchewan: Our Dream Is That Our Peoples Will One Day Be Clearly Recognised as Nations. Calgary, University of Calgary Press.

Clammer, John, Sylvie Poirier, et Eric Schwimmer (dir.), 2004. Figured Worlds: Ontological Obstacles in Intercultural Relations. Toronto, University of Toronto Press.

Chakrabarty, Dipesh, 2009. Provincialiser l'Europe. La pensée postcoloniale et la différence historique. Paris, Éditions Amsterdam.

Coulthard, Glen Sean, 2014. Red Skin, White Masks: Rejecting the Colonial Politics of Recognition. Minneapolis, University of Minnesota Press.

Coyle, Michael, 2017. "As Long as the Sun Shines: Recognizing that Treaties Were Intended to Last ". In John Borrows et Michael Coyle (dir.), The Right Relationship: Reimagining the Implementation of Historical Treaties, p. 39-69. Toronto, University of Toronto Press.

Dempsey, Hugh A., et L. James Dempsey, 2014. « The Blackfoot Nation ». In C. Roderick Wilson et Christopher Fletcher (dir.), Native Peoples: The Canadian Experience, Fourth Edition, p. 303-327. Don Mills, ON, Oxford University Press.

Depelteau, Julie, 2019. Nitaskinan, territoire. Analyse comparative des discours des représentants politiques des Atikamekw Nehirowisiwok et des gouvernements coloniaux, 1973-2004. Thèse de doctorat, Université d'Ottawa.

Davis, Lynne (dir.), 2010. Alliances: Re/envisioning Indigenous-non-Indigenous Relationships. Toronto, Toronto University Press.

Dussart, Françoise, et Sylvie Poirier (dir.), 2017. Entangled Territorialities: Negotiating Indigenous Lands in Australia and Canada. Toronto, University of Toronto Press.

Eisenberg, Avigail, Jeremy Webber, Glen Coulthard, et Andrée Boisselle, 2014. Recognition versus Selfdetermination: Dilemmas of Emancipatory Politics. Vancouver, University of British Columbia Press.

Hébert, Martin, 2019. « Participation autochtone à la gestion des territoires et ressources naturelles au Québec : De l'extension des droits à la production de futurs ». In Didier Busca et Nathalie Lewis (dir.), Penser le gouvernement des ressources naturelles, p. 371-394. Québec, Presses de l'Université Laval.
Hill, Susan M., 2008. « Traveling Down the River of Life Together in Peace and Friendship, Forever: Haudenosaunee Land Ethics and Treaty Agreement as the Basis for Restructuring the Relationship with the British Crown ". In Leanne Betasamosake Simpson (dir.), Lighting the Eighth Fire: The Liberation, Resurgence, and Protection of Indigenous Nations, p. 23-45. Winnipeg, ARP Books.

Jullien, François, 2009. " Respecter la diversité culturelle ». Débat, 153 (1) : 157-162. https://doi.org/10.3917/deba.153.0157

Kapesh, An Antane, 1976. Eukuan nin matshi-manitu innushkueu / Je suis une maudite Sauvagesse. Montréal, Léméac.

- - 1979. Tante nana etutamin nitassi / Qu'as-tu fait de mon pays? Montréal, Éditions Impossible.

Manuel, Arthur, et Ronald Derrickson, 2017. The Reconciliation Manifesto: Recovering the Land, Rebuilding the Economy. Toronto, James Lorimer and Company Ltd. Publishers.

Miller, James Rodger, 2009. Compact, Contract, Covenant. Aboriginal Treaty-Making in Canada. Toronto, University of Toronto Press.

Mills, Aaron / Waabishki ma'iingan, 2017. « What Is a Treaty? On Contract and Mutual Aid ». In John Borrows, et Michael Coyle (dir.), The Right Relationship: Reimagining the Implementation of Historical Treaties, p. 218-244. Toronto, University of Toronto Press.

Morales, Sarah, 2017. " (Re)defining “Good Faith" through Snuw'uyulh ». In John Borrows et Michael Coyle (dir.), The Right Relationship: Reimagining the Implementation of Historical Treaties, p. 277-302. Toronto, University of Toronto Press.

Nadasdy, Paul, 2003. Hunters and Bureaucrats: Power, Knowledge, and Aboriginal-State Relations in the Southwest Yukon. Vancouver, University of British Columbia Press.

_, 2017. Sovereignty's Entailments: First Nation State Formation in the Yukon. Toronto, University of Toronto Press.

Papillon, Martin, et Audrey Lord, 2013. « Les traités modernes : Vers une nouvelle relation ? ». In Alain Beaulieu, Stéphan Gervais et Martin Papillon (dir.), Les Autochtones et le Québec. Des premiers contacts au Plan Nord, p. 343-362. Montréal, Les Presses de l'Université de Montréal.

Pasternak, Shiri, 2017. Grounded Authority: The Algonquins of Barriere Lake against the State. Minneapolis, University of Minnesota Press.

Poirier, Sylvie, 2010. « Introduction. Imagination culturelle et politique dans les communautés autochtones au Canada et en Australie ". Anthropologica, 52 (1) : 3-8.

_ 2013. « The Dynamic Reproduction of HunterGatherers' Ontologies and Values ". In Janice Boddy et Michael Lambek (dir.), A Companion to the Anthropology of Religion, p. 50-68. Oxford, Wiley Blackwell.

Pratt, Angela, 2004. "Treaties vs. Terra Nullius: "Reconciliation," Treaty-Making and Indigenous Sovereignty in Australia and Canada ". Indigenous Law Journal, 3 : 43-60.

Reddekopp, G. Neil, 1997. « The Treaty Land Entitlement Claim of the Bigstone Cree Nation.” Alberta Aboriginal Affairs and Northern Development. 
Roy, Jean-Olivier, 2018. «Identité et citoyenneté : Le fédéralisme de traités à l'ère (post)coloniale ». Conférence présentée au Colloque annuel du Ciéra. Québec.

$\ldots, 2019$. « Renvoi relatif à la sécession du Québec et dialogue constitutionnel : Quelles ouvertures pour les Autochtones ? ». In Félix Mathieu et Dave Guénette (dir.), Ré-imaginer le Canada-Vers un état multinational, p. 173-195. Québec, Presses de l'Université Laval.

Samson, Colin, 2016. " Canada's Strategy of Dispossession: Aboriginal Land and Rights Cessions in Comprehensive Land Claims ". Canadian Journal of Law and Society / Revue Canadienne Droit et Société, 31 (1) : 87-110. https:// doi.org/10.1017/cls.2016.2

Sapir, Edward, 1921. Language: An Introduction to the Study of Speech. New York, Harcourt, Brace, and Company.

Scott, Colin, 2017. « The Endurance of Relational Ontology: Encounters between Eeyouch and Sport Hunters ». In Françoise Dussart et Sylvie Poirier (dir.), Entangled Territorialities: Negotiating Indigenous Lands in Australia and Canada, p. 51-69. Toronto, University of Toronto Press.

Simpson, Leanne Betasamosake (dir.), 2008. Lighting the Eighth Fire: The Liberation, Resurgence, and Protection of Indigenous Nations. Winnipeg, ARP Books.

— 2015. « The Place Where We All Live and Work Together: A Gendered Analysis of "Sovereignty" ». In Stephanie Nohelani Teves, Andrea Smith et Michelle H. Raheja (dir.), Native Studies Keywords, p. 18-24. Tucson, AZ, The University of Arizona Press.

Starblanket, Gina, et Heidi Kiiwetinepinesiik Stark, 2018. " Towards a Relational Paradigm - Four Points for Consideration: Knowledge, Gender, Land, and Modernity ». In Michael Asch, John Borrows et James Tully (dir.), Resurgence and Reconciliation: IndigenousSettler Relations and Earth Teachings, p. 175-207.

Toronto, University of Toronto Press.
Treaty 7 Tribal Council, Walter Hildebrandt, Sarah Carter, et Dorothy First Rider. 1996. The True Spirit and Original Intent of Treaty \%. Montréal, McGill-Queen's University Press.

Tully, James, 2008. Public Philosophy in a New Key. Volume I: Democracy and Civic Freedom. Cambridge, Cambridge University Press. , 2016. «Deparochializing Political Theory and Beyond: A Dialogue Approach to Comparative Political Thought ». Journal of World Philosophies, 1 : 51-74.

Vincent, Sylvie, 2016. " "Chevauchements" territoriaux : Ou comment l'ignorance du droit coutumier algonquins permet de créer de faux problèmes ". Recherches Amérindiennes au Québec, 46 (2-3) : 91-103. https://doi. org/10.7202/1040438ar

Westman, Clinton N., 2016. « Aboriginal Subsistence Practices in an "Isolated" Region of Northern Alberta ". In Dean Bavington, James Murton et Carly Dokis (dir.), Subsistence Under Capitalism: Historical and Contemporary Perspectives, p. 162-194. Montréal, McGillQueen's University Press.

Westman, Clinton N., 2017a. « Negotiating and Implementing Treaty Eight in the Athabasca District/Northern Alberta, Canada: 1899-2017 ». Conférence présentée dans le cadre du Colloque annuel de CASCA. Ottawa.

Westman, Clinton N. 2017b. « Cultural Politics of Land and Animals in Treaty Eight Territory (Northern Alberta, Canada) ». In Françoise Dussart et Sylvie Poirier (dir), Entangled Territorialities: Negotiating Indigenous Lands in Australia and Canada, p. 117-139. Toronto, University of Toronto Press.

Wolfart, H. Christoph, et Freda Ahenakew. 1998. The Student's Dictionary of Literary Plains Cree: Based on Contemporary Texts. Winnipeg, Algonquian and Iroquoian Linguistics. 